\title{
Identification of antenatal depression in obstetric care
}

\author{
Judith Alder • Nadine Fink • Corinne Urech • \\ Irene Hösli · Johannes Bitzer
}

Received: 3 September 2010/Accepted: 25 February 2011/Published online: 19 March 2011

(c) Springer-Verlag 2011

\begin{abstract}
Purpose Detection rates of depression in obstetric care are generally low, and many women remain undiagnosed and do not receive adequate support. In many obstetric settings, screening tools for depression are not applied routinely and there is a great need to sensitize health care professionals for the patient at risk for enhanced levels of depression. The present study aimed at identifying commonly assessed patient characteristics that are associated with antenatal depression.

Methods One hundred and thirty seven women were screened using the Edinburgh Postnatal Depression Scale (EPDS) at the beginning of the second trimester at the outpatient department of a Tertiary University Hospital. Women were identified as at high risk for depression if scores were above a cut-off score of twelve. Obstetric history and outcome were extracted from patient files after delivery.

Results Twenty one percent of the sample screened as depression positive. Logistic regression with backwards elimination showed that the triad of nausea during pregnancy, reports of (premature) contractions and
\end{abstract}

J. Alder $(\bowtie) \cdot$ C. Urech · I. Hösli · J. Bitzer

University Women's Hospital, University of Basel,

Spitalstrasse 21, 4031 Basel, Switzerland

e-mail: jalder@uhbs.ch

N. Fink

Children's Hospital, Harvard Medical School, Boston,

Hunnewell 2, Rm HU-260.2, 300 Longwood Ave,

Boston, MA 02115, USA

N. Fink

Department of Child and Adolescent Psychiatry and Psychotherapy, University of Basel, Schaffhauserrheinweg 55, 4058 Basel, Switzerland consumption of analgesics during pregnancy significantly predicted high depression scores with a positive predictive value of $84.3 \%$. The relative risk for a depressed pregnant woman to regularly take analgesics during pregnancy was fourfold higher than for non-depressed women.

Conclusions If depression screening is not part of routine prenatal care, systematic assessment of depression should be targeted for patients presenting with the markers identified in this study.

Keywords Antenatal depression - Screening · Patient characteristics · Nausea - Analgesics

\section{Introduction}

While in the context of reproduction for many years, the focus has been on postnatal depression, studies of the past decade have shown that the incidence of postnatal depression is lower than expected and that the majority of cases of postnatal depression are preceded by antenatal depression [1]. A recent meta-analysis [2] of 21 studies on the prevalence of depression during pregnancy differentiates between questionnaire-based studies and studies that determine the prevalence of depression by structured interviews. The overall rate of depression was 7.4, 12.8 and $12 \%$ for each trimester, respectively, with comparable numbers that have been found for Switzerland [3]. The prevalence rates detected with structured interviews or the Edinburgh postnatal depression scale (EPDS) [4] did not differ significantly.

Despite the fact that according to these numbers, at least every tenth pregnant woman presenting for regular checkups during pregnancy will be depressed, especially during pregnancy, detection rates of depressive illness are 
low: only $26 \%$ of patients who screened positive for a psychiatric illness was recognized as having a mood or anxiety disorder and detection rate for depressive disorders was even lower [5] which is comparable to other nonpsychiatric medical disciplines [6]. As Kammerer et al. [3] point out, it has been disregarded how gestation-associated physiological and psychosocial changes may confound the diagnosis of depression and thus contribute to the low detection rates in prenatal care. In addition, clinicians generally do not address mood states at prenatal visits. The legitimate focus of attention in antenatal care lies on the optimal course of pregnancy, physical wellbeing of the pregnant woman and foetal condition. However, with many recent studies pointing to a negative impact of antenatal mental health problems on course of pregnancy, foetal and neonatal outcome (for review see [7]), the assessment of psychological state becomes relevant for obstetric care as well. In addition, independent of its influence on outcome, depression is associated with negative health behaviour [8] and adversely affects the mother's functional state and quality of life, and is a relevant illness which needs to be treated appropriately.

Depression during pregnancy has been associated with obstetric complications, and adverse foetal and neonatal parameters of wellbeing. Depressed women report more somatic symptoms, have more visits to the obstetrician, and receive more pain relief during labour [9-13]. While a global score of obstetric complications appears to be enhanced in depressed patients [14], there is still controversy about the impact of depression on the development of specific pregnancy associated diseases such as preeclampsia $[10,15,16]$. In addition, controversies exist on the effect of depressive symptoms on mode of delivery [10, 12, 17, 18]. Moreover, foetuses of depressed mothers seem to be more active in general and more reactive to a standardized maternal stress condition [14, 19-21]. In addition, a negative impact of antenatal depression on birth weight and gestational age with some controversies [22-26], and higher admission rates to Neonatal Intensive Care Unit NICU [12, 27] have been reported.

These results point to the importance of an early detection of women with an enhanced risk for depression. These assessments may take the form of a brief questionnaire or a structured psychosocial interview. The EPDS is the most widely accepted screening scale, has been translated into different languages, and is used internationally in the perinatal period. While having been developed to screen for postnatal depression in the primary care setting, validation studies have shown its suitability to screen for depression in the antenatal period as well [28, 29]. Although it appears simple to use, training in administering and scoring the scale, giving women appropriate feedback, and understanding its limitations are important. Several concerns have been discussed for the implementation of screening programs in antenatal care such as false positive and false negative results and the corresponding consequences for the pregnant women and the health care professionals, the risk of stigmatization or the lack of treatment resources when depression is confirmed. However, as Buist et al. [30] point out, the alternative of not identifying distressed women is not satisfactory either and it is better to identify distress and attempt to deal with it as optimal as possible than to deny its existence and suffer the potential longterm consequences. Yet, the implementation of systematic screening in clinical routine is often jeopardized by institutional and intrapersonal factors such as time constraints, limited personnel or lack of skills. In this context, it may be essential to sensitize health care professionals to characteristics of pregnant women at risk for antenatal distress and enhance their skills in identifying those women where the application of a screening questionnaire is suggestive. As suggested by Jesse and Graham [31] one or two questions can be used to decide, whether the application of a screening tool can be reasonable.

Another approach is by identifying socio-demographic factors which are known to be associated with antenatal depression. Some perinatal studies have identified younger age, lower socioeconomic status, lower levels of education [26, 32], belonging to a minority group [5, 33] and not being married [32, 34] to be associated with states of depression, while others have not found some of these associations [3, 26, 35]. In addition, some studies point to differences in antenatal care between women with high levels of depression and healthy women with more patient complaints on somatic symptoms, mostly abdominal pain [13] and more prescriptions of antibiotics for the depressed [32]. Not much is known on other factors which belong to routine clinical assessment during antenatal care and their association to levels of depression.

While obstetricians are not trained often to systematically assess psychological symptoms during pregnancy by use of specific questions or validated questionnaires, the recognition of specific characteristics of depressed pregnant women can facilitate the identification of women with enhanced levels of depression in antenatal care.

Therefore, the aim of this study is to detect a profile of depressed pregnant women considering medical information and patient characteristics which are routinely being assessed during antenatal care. For this purpose, women with enhanced levels of depression according to the EPDS will be compared to asymptomatic women with regard to regularly assessed clinical characteristics in order to identify those factors which are predictive for antenatal depressive symptoms. 


\section{Methods}

Study population

The present retrospective case-control study is part of a placebo-controlled trial on the effectiveness of light therapy for the treatment of antenatal depression. Germanspeaking women attending routine pregnancy care at the beginning of the second trimester at University Women's Hospital of Basel, Switzerland, after being informed and consenting to participation, were screened for depression. Patients with depression scores $>12$ in the Edinburgh Postnatal Depression Scale (EPDS) according to the German validation of the questionnaire [36] are identified as probable cases of depression (index group) and are compared to the group of pregnant women with scores below the cut-off score (controls). During the consultation, the obstetrician referred to the results of the EPDS and discussed with high scoring patients the subsequent procedures. If the patient agreed, she was referred to the light therapy study, where depression was confirmed by structured clinical interview (SCID). The present data analysis includes results from this screening procedure. To guarantee a high and comparable standard of data quality between depressed and non-depressed patients, we only considered records of women, who had all pregnancy controls starting from the first trimester to delivery at the clinic. In total, 137 patients were enrolled.

\section{Measures}

\section{Edinburgh postnatal depression scale (EPDS)}

Symptoms of depression were assessed by the EPDS [4, 36], a 10-item screening tool providing an indication of symptom severity. Items are rated on a four-point Likert scale. The EPDS is the only screening tool for validating depression also for the perinatal period. To identify women with probable major depression in the postpartum a cut-off score of 13 or more has been found useful across various studies, while the use of a score of 10 or more has been recommended to identify women at risk of postnatal minor depression or increase in the sensitivity [29]. For the detection of patients at risk of fulfilling the criteria of major depression during pregnancy, a higher score of 15 or more and for those with risk of antenatal minor depression a score of 13 or more has been recommended based upon published validation studies [29]. For the present analysis we decided to evaluate clinical characteristics of women with enhanced levels of depression and not only those at risk for major depression, and therefore used the cut-off score of 13 or more. The identification of these women during antenatal care and provision of support might not only prevent the exacerbation of their depressive symptoms but also might be beneficial for obstetric and neonatal outcome.

\section{Pregnancy data}

Information on course of pregnancy, complications, delivery and neonatal outcome were extracted from patient records and birth reports.

To identify a profile of depression during pregnancy, the following background variables were selected: age, parity, pre-pregnancy BMI, intake of medication during pregnancy, smoking, provenience, and subjectively reported contractions. These variables were chosen because they are part of standard history taking during prenatal checkups, while during prenatal care very often information on other risk factors such as family affective disorder history, past psychiatric history, current levels of stress and resources such as social support are not assessed.

\section{Statistical analysis}

Using logistic regression analysis, we determined the impact of the selected background variables on the dependent variable high depression scores (defined as EPDS-score $>12$ ). The variables age, parity, origin (categorical: (1) Northern and Western Europe, North America and (2) Southern, Eastern Europe, South America, Africa, Asia), pre-pregnancy BMI, patient reports of premature contractions before pregnancy week 37 (categorical: yes/ no), nausea (categorical: yes/no), smoking (categorical: yes/no) and consumption of medication entered as candidate predictors. Backward elimination was conducted with probability of inclusion set at 0.05 and exclusion set at 0.10 . Consumption of medication was categorized as none, analgesics and other and no consumption of medication served as the reference for the latter two. The overall model fit was tested with the likelihood ratio test, and Nagelkerke's $R^{2}$ statistics are reported as well considering the rather low number of depressed patients. Percentage of overall correct predictions and specificity and sensitivity values for the predictors remaining in the model were calculated. Data were analysed using SPSS 16.

\section{Results}

Table 1 displays main characteristics of the studied sample and compares women with EDPS scores of 13 or more to those with lower scores on socio-demographic variables. Mean age of patients was 30 years and mean parity was 1.64 , reflecting the average number of children/woman in Switzerland. There were no differences between women 
Table 1 Socio-demographic characteristics of the total sample and women with high and low EPDS scores

$+p<0.1$

${ }^{a}$ Reported as mean (SD)

b Reported as number (\%)

\begin{tabular}{lccr}
\hline & \multicolumn{1}{l}{ Total } & EPDS 13 or more & EPDS below 13 \\
\hline Age $^{\mathrm{a}}$ & $30.11(6.22)$ & $29.86(6.51)$ & $30.18(6.17)$ \\
Parity $^{\mathrm{a}}$ & $1.64(0.74)$ & $1.66(0.72)$ & $1.64(0.74)$ \\
Weight of child $^{\mathrm{a}}(\mathrm{g})$ & $3,312.66(492.05)$ & $3,359.81(464.24)$ & $3,299.90(500.85)$ \\
Length of gestation $^{\mathrm{a}}$ & $39.59(1.78)$ & $40.20(1.08)$ & $39.43(1.895)^{+}$ \\
Migration background $^{\mathrm{b}}$ & $55(40.1)$ & $16(55.2)$ & $39(36.1)^{+}$ \\
\hline
\end{tabular}

who scored 13 or above with regard to age, parity, and birth weight, however there was a tendency for longer gestations in this group (40.2 vs. 39.6 weeks) and for having a migration background. As shown in Table 2, women with an EPDS cut-off score of 13 or above differed significantly with regard to several study variables. They reported more premature contractions, more nausea during pregnancy and a higher intake of medication and, more specifically, analgesics. In contrast to many other studies on the association of depressive symptoms and unhealthier life style, the high scorers in this group did not differ with regard to smoking behaviour and BMI.

In Table 3, the results of the logistic regression analysis for the identification of patient characteristics predictive for depression are shown. In a first step, all of the variables were included and premature contractions $(\mathrm{OR}=4.2$, $p<0.05)$, nausea ( $\mathrm{OR}=3.7, p<0.05)$ and analgesics $(\mathrm{OR}=8.4, p<0.01)$ resulted to be predictive for EPDS scores of 13 or more. These three variables report of premature contractions, nausea, and intake of analgesics remained in the final model, which resulted highly significant $\left(\chi^{2}=30.87, d f=4, p<0.001\right)$ with a Nagelkerke's $R^{2}=0.38$. The total amount of correct predictions of depression for the three variables reported premature contractions, nausea and consumption of analgesics was $84.3 \%$, with a specificity of 0.95 and sensitivity of 0.44 . The relative risk (RR) for patients with depression scores above the EPDS score of 12 to report premature contractions was $R R=2.44$, to suffer from nausea or vomiting $\mathrm{RR}=1.75$ and to consume analgesics during pregnancy was $\mathrm{RR}=4.30$.

\section{Discussion}

Using an EPDS-cut-off score of 13 or more we found enhanced levels of depression in $21.1 \%$ of our sample. While being indicative for the presence of a minor depression, we found scores of 15 and above in $10.9 \%$ of the study population. Thus, the observed rate of probable major depression is comparable to other studies [27], while, in line with other data, minor depression affected about every fifth pregnant woman [37]. With the growing evidence that clinical and subclinical antenatal depression negatively affects the course of pregnancy, foetal development and neonatal adaptation [7], there is a great need to identify women at risk. As there are still only a few countries and health care systems which have introduced screening for depression in standard obstetric care, it is important to sensitize health care professionals to probable signs of antenatal depression. The present study, therefore aimed at the evaluation of variables which are part of standard obstetric history taking in most settings, and at investigating their association to antenatal depression.

Including socio-demographic and obstetric parameters, those variables which correctly identified $84.3 \%$ of the women with an EPDS score of 13 or more, were subjective report of premature contractions, nausea and consumption of analgesics with the latter being associated with a fourfold enhanced relative risk for depression. Age, parity, health behaviour such as smoking and BMI and having a migration background were not related to antenatal depression. The sample of our present study, however, included only German speaking women. Therefore,
Table 2 Comparison of woman with high and low EPDS sum scores

\footnotetext{
$* * p<0.01$

${ }^{a}$ Reported as number (\%)

b Reported as mean (SD)
}

\begin{tabular}{lccc}
\hline & Total & EPDS 13 or more & EPDS below 13 \\
\hline Subjectively reported premature contractions $^{\mathrm{a}}$ & $46(33.6)$ & $18(72)$ & $29(26.9)^{* *}$ \\
Smoking during pregnancy $^{\mathrm{a}}$ & $37(27)$ & $9(33.3)$ & $28(26.2)$ \\
Reported nausea/vomiting $^{\mathrm{a}}$ & $71(51.8)$ & $22(81.5)$ & $49(45.4)^{* *}$ \\
Medication during pregnancy $^{\mathrm{a}}$ & $60(43.8)$ & $19(65.5)$ & $41(38)^{* *}$ \\
Analgesics $^{\mathrm{a}}$ & $26(19)$ & $14(48.3)$ & $12(11.1)^{* *}$ \\
Prepregnancy BMI $^{\mathrm{a}}\left(\mathrm{kg} / \mathrm{m}^{2}\right)$ & $23.09(4.83)$ & $22.34(2.46)$ & $23.33(5.36)$ \\
EPDS sum score $^{\mathrm{b}}$ & $7.12(5.76)$ & $15.97(3.17)$ & $4.75(3.56)^{* *}$ \\
EPDS $>15^{\mathrm{a}}$ & $15(10.9)$ & & \\
\hline
\end{tabular}


Table 3 Logistic regression analysis with backwards elimination on background variables predicting high levels of depression (first and final step after backwards elimination)

\begin{tabular}{llll}
\hline & $B$ & OR & CI \\
\hline First step & & & \\
Age & 0.04 & 1.04 & $0.93-1.17$ \\
Migration background & 0.64 & 1.9 & $0.51-7.10$ \\
Parity & -0.47 & 0.62 & $0.23-1.69$ \\
BMI & -0.09 & 0.91 & $0.76-1.09$ \\
Smoking & 0.48 & 1.62 & $0.43-6.09$ \\
Premature contractions & 1.25 & 3.49 & $1.09-11.22^{*}$ \\
Nausea & 1.24 & 3.47 & $0.96-12.53^{+}$ \\
Medication & & & \\
$\quad$ No & Reference & & \\
$\quad$ Analgesics & 2.09 & 8.12 & $1.70-38.834^{* *}$ \\
$\quad$ Other medication & -0.34 & 0.72 & $0.17-3.06$ \\
Final step & & & \\
Premature contractions & 1.28 & 3.61 & $1.17-11.08^{*}$ \\
Nausea & 1.21 & 3.34 & $0.98-11.39^{+}$ \\
Medication & & & \\
$\quad$ No & & & \\
$\quad$ Analgesics & Reference & & \\
$\quad$ Other medication & 1.91 & 6.73 & $1.59-12.54^{* *}$ \\
\hline$+p<0.1$ & -0.34 & 0.71 & $0.17-2.93$ \\
$*$ * & & & \\
* 0.05 & & &
\end{tabular}

especially those migrant women with a high degree of stress due to socioeconomic concerns such as acculturation problems, higher unemployment rates and for whom differences in pregnancy outcome have been reported before [38], did not participate in the study. The finding that the other demographic factors were not associated with higher rates of depression, contrasts with some studies who have found higher levels of depression in younger women and women from minority groups $[5,27,32]$. On the other hand, our results are in line with data from other groups who have found more complaints about abdominal pain in the depressed [13]. The psychosomatic nature of the depression-associated factors in the current study points to a high degree of somatization of depression during pregnancy or its presentation with somatic symptoms and is in support of other findings [13, 39]. It is, in addition, a finding with important clinical implications as the presentation of somatic symptoms may mask the underlying symptoms of depressions and lead to inadequate interventions. Prenatal depression therefore often presents as a "silent" form of depression, it may be difficult to differentiate the depressive symptoms from general mood swings due to hormonal alterations and complaints about pregnancy-associated physical changes. However, as shown by the results of this study, practitioners must be aware of a possible overlap of general complaints and depressive symptoms, as prenatal depression, can have adverse effects if left untreated.

Also, it remains to be determined, if in depressed pregnant women the processing of proprioceptive signals itself or the cognitive attribution of perceived signals are altered and thus depressogenic.

The specificity of the correlates of antenatal depression found in this study is high, therefore being a reliable way to identify those pregnant women with a chance of being depressed. However, the sensitivity is too low in order to only rely on the identified correlates considering that more than half of the truly depressed patients will not be identified. The findings thus indicate that the probability of depression is high if a woman presents with nausea reports of premature contractions and intake of analgesics; however, depression can also be present without these symptoms.

Optimally, screening for depression should therefore be implemented in routine prenatal care and it has been shown that it is well accepted in this population [40]. As stated by the American College of Obstetricians and Gynecologists ACOG, screening should also include depression-associated risk factors, such as lower use of health care, housing problems, violence and inadequate health behaviours [41]. Also, there is general consensus that screening for depression in prenatal care should be delivered systematically including predefined referral pathways as it is unlikely that antenatal psychosocial assessment alone already leads to improved mental health outcomes [42]. An interdisciplinary approach and communication training for staff members are additional features of successful screening concepts [41, 43]. Communication skills to sensitively address the psychological state of a pregnant woman are especially important in systems without implemented screening. As such, pregnant women conspicuous with the symptoms found in the present study need to be sensitively questioned about their psychological wellbeing, asking for general wellbeing, current stressors and concerns and preexisting or recent mood swings and sadness.

Alternatively, the two screening questions, "Have you lately often been sad and depressed" and "Have you had a loss in pleasurable activities", have shown a sensitivity of $92 \%$ and specificity of $52 \%$ to identify patients suffering from depression during pregnancy [31].

Several limitations of the present study need to be discussed. Besides modest sample size, at the time of the study the EPDS was only available to us in German. Thus, an important risk group, women who recently migrated to Switzerland without sufficient language skills, were not included. In addition, while during the observation period all women who presented for routine checkup at gestational 
week 15 were asked to participate, some women were not included due to time constraints by the staff. Also, women who presented later or had their checkups outside of the hospital were not included either. Thus, while the results cannot be generalized, the validity of the obstetric information is good and comparable within the study population. Nevertheless, the information assessed during routine prenatal care did not include other factors known to be associated to antenatal depression such as socioeconomic status or the previous history of psychopathology. While being a limitation for comprehensive psychosocial assessment, it still reflects the clinical reality of many prenatal care services.

\section{Conclusions}

From the current literature and present study, the following conclusions can be drawn. Health care providers, specifically those in obstetric care, should be aware of (1) the frequency of depression in pregnant women. Being as prevalent as in non-pregnant state, prenatal depression is under recognized in prenatal care [5] and there is a great need to sensitize obstetricians for the recognition of patients with depression during pregnancy. (2) Obstetricians should be aware of signs and symptoms of depression and appropriate screening methods. If depression screening cannot be implemented in the obstetric routine, pregnant women presenting with the triad nausea, contractions and consumption of analgesics should be carefully screened and history and current levels of depression should be assessed unconditionally. (3) The health risks of undetected and untreated depression for the mother and growing foetus are considerable. For example, depression is associated with elevated risk for preterm delivery, low birth weight and intrauterine growth retardation comparable to other obstetric risk factors, which are controlled for as part of standard routine care (e.g., blood pressure, proteinuria) [44]. The awareness of these associations might improve adherence to screening for depression in prenatal care.

\section{Conflict of interest None.}

\section{References}

1. Heron J, O'Connor TG, Evans J, Golding J, Glover V (2004) The course of anxiety and depression through pregnancy and the postpartum in a community sample. J Affect Disord 80:65-73

2. Bennett HA, Einarson A, Taddio A, Koren G, Einarson TR (2004) Prevalence of depression during pregnancy: systematic review. Obstet Gynecol 103:698-709

3. Kammerer M, Marks MN, Pinard C, Taylor A, von Castelberg B, Kunzli H, Glover V (2009) Symptoms associated with the DSM
IV diagnosis of depression in pregnancy and post partum. Arch Womens Ment Health 12:135-141

4. Cox JL, Holden JM, Sagovsky R (1987) Detection of postnatal depression. Development of the 10-item Edinburgh postnatal depression scale. Br J Psychiatry 150:782-786

5. Smith MV, Rosenheck RA, Cavaleri MA, Howell HB, Poschman K, Yonkers KA (2004) Screening for and detection of depression, panic disorder, and PTSD in public-sector obstetric clinics. Psychiatry Serv 55:407-414

6. Cepoiu, M, McCusker J, Cole MG, Sewitch M, Belzile E, Ciampi A (2007) Recognition of depression by non-psychiatric physicians-a systematic literature review and meta-analysis. J Gen Intern Med

7. Alder J, Fink N, Bitzer J, Hosli I, Holzgreve W (2007) Depression and anxiety during pregnancy: a risk factor for obstetric, fetal and neonatal outcome? A critical review of the literature. J Matern Fetal Neonatal Med 20:189-209

8. Zuckerman B, Amaro H, Bauchner H, Cabral H (1989) Depressive symptoms during pregnancy: relationship to poor health behaviors. Am J Obstet Gynecol 160:1107-1111

9. Andersson L, Sundstrom-Poromaa I, Bixo M, Wulff M, Bondestam K, aStrom M (2003) Point prevalence of psychiatric disorders during the second trimester of pregnancy: a population-based study. Am J Obstet Gynecol 189:148-154

10. Andersson L, Sundstrom-Poromaa I, Wulff M, Astrom M, Bixo M (2004) Implications of antenatal depression and anxiety for obstetric outcome. Obstet Gynecol 104:467-476

11. Larsson C, Sydsjo G, Josefsson A (2004) Health, sociodemographic data, and pregnancy outcome in women with antepartum depressive symptoms. Obstet Gynecol 104:459-466

12. Chung TK, Lau TK, Yip AS, Chiu HF, Lee DT (2001) Antepartum depressive symptomatology is associated with adverse obstetric and neonatal outcomes. Psychosom Med 63:830-834

13. Kelly RH, Russo J, Katon W (2001) Somatic complaints among pregnant women cared for in obstetrics: normal pregnancy or depressive and anxiety symptom amplification revisited? Gen Hosp Psychiatry 23:107-113

14. Field T, Miguel AD, Dieter J, Hernandez-Reif M, Schanberg S, Kuhn C, Yando R, Bendell D (2001) Depressed withdrawn and intrusive mothers' effects on their fetuses and neonates. Infant BehavDev 24:27-39

15. Kurki T, Hiilesmaa V, Raitasalo R, Mattila H, Ylikorkala $O$ (2000) Depression and anxiety in early pregnancy and risk for preeclampsia. Obstet Gynecol 95:487-490

16. Qiu C, Sanchez SE, Lam N, Garcia P, Williams MA (2007) Associations of depression and depressive symptoms with preeclampsia: results from a Peruvian case-control study. BMC Womens Health 7:15

17. Perkin MR, Bland JM, Peacock JL, Anderson HR (1993) The effect of anxiety and depression during pregnancy on obstetric complications. Br J Obstet Gynaecol 100:629-634

18. Wu J, Viguera A, Riley L, Cohen L, Ecker J (2002) Mood disturbance in pregnancy and the mode of delivery. Am J Obstet Gynecol 187:864-867

19. Field T, Diego M, Hernandez-Reif M, Schanberg S, Kuhn C, Yando R, Bendell D (2003) Pregnancy anxiety and comorbid depression and anger: effects on the fetus and neonate. Depress Anxiety 17:140-151

20. Dieter JN, Field T, Hernandez-Reif M, Jones NA, Lecanuet JP, Salman FA, Redzepi M (2001) Maternal depression and increased fetal activity. J Obstet Gynaecol 21:468-473

21. Monk C, Sloan RP, Myers MM, Ellman L, Werner E, Jeon J, Tager F, Fifer WP (2004) Fetal heart rate reactivity differs by women's psychiatric status: an early marker for developmental risk? J Am Acad Child Adolesc Psychiatry 43:283-290 
22. Steer RA, Scholl TO, Hediger ML, Fischer RL (1992) Selfreported depression and negative pregnancy outcomes. J Clin Epidemiol 45:1093-1099

23. Hoffman S, Hatch MC (2000) Depressive symptomatology during pregnancy: evidence for an association with decreased fetal growth in pregnancies of lower social class women. Health Psychol 19:535-543

24. Orr ST, James SA, Blackmore Prince C (2002) Maternal prenatal depressive symptoms and spontaneous preterm births among African-American women in Baltimore, Maryland. Am J Epidemiol 156:797-802

25. Andersson L, Sundstrom-Poromaa I, Wulff M, Astrom M, Bixo M (2004) Neonatal outcome following maternal antenatal depression and anxiety: a population-based study. Am J Epidemiol 159:872-881

26. Dayan J, Creveuil C, Marks MN, Conroy S, Herlicoviez M, Dreyfus M, Tordjman S (2006) Prenatal depression, prenatal anxiety, and spontaneous preterm birth: a prospective cohort study among women with early and regular care. Psychosom Med 68:938-946

27. Misri S, Oberlander TF, Fairbrother N, Carter D, Ryan D, Kuan AJ, Reebye P (2004) Relation between prenatal maternal mood and anxiety and neonatal health. Can J Psychiatry 49:684-689

28. Murray L, Carothers AD (1990) The validation of the Edinburgh Post-natal Depression Scale on a community sample. Br J Psychiatry 157:288-290

29. Matthey S, Henshaw C, Elliott S, Barnett B (2006) Variability in use of cut-off scores and formats on the Edinburgh postnatal depression scale: implications for clinical and research practice. Arch Womens Ment Health 9:309-315

30. Buist AE, Barnett BE, Milgrom J, Pope S, Condon JT, Ellwood DA, Boyce PM, Austin MP, Hayes BA (2002) To screen or not to screen - that is the question in perinatal depression. Med J Aust 177(Suppl):S101-105

31. Jesse DE, Graham M (2005) Are you often sad and depressed? Brief measures to identify women at risk for depression in pregnancy. Am J Matern Child Nurs 30:40-45

32. Field T, Hernandez-Reif M, Diego M (2006) Risk factors and stress variables that differentiate depressed from nondepressed pregnant women. Infant Behav Dev 29:169-174

33. Zayas LH, Cunningham M, McKee MD, Jankowski KR (2002) Depression and negative life events among pregnant African-
American and Hispanic women. Women's Health Issues $12: 16-22$

34. Lindgren K (2001) Relationships among maternal-fetal attachment, prenatal depression, and health practices in pregnancy. Res Nurs Health 24:203-217

35. Diego MA, Jones NA, Field T, Hernandez-Reif M, Schanberg S, Kuhn C, Gonzalez-Garcia A (2006) Maternal psychological distress, prenatal cortisol, and fetal weight. Psychosom Med 68:747-753

36. Bergant AM, Nguyen T, Heim K, Ulmer H, Dapunt O (1998) German language version and validation of the Edinburgh postnatal depression scale. Dtsch Med Wochenschr 123:35-40

37. Flynn HA, Walton MA, Chermack ST, Cunningham RM, Marcus SM (2007) Brief detection and co-occurrence of violence, depression and alcohol risk in prenatal care settings. Arch Women's Ment Health 10:155-161

38. Alder J, Fink N, Lapaire O, Urech C, Meyer A, Bitzer J, Hosli I, Holzgreve W (2008) The effect of migration background on obstetric performance in Switzerland. Eur J Contracept Reprod Health Care 13:103-108

39. Seng JS, Low LK, Ben-Ami D, Liberzon I (2005) Cortisol level and perinatal outcome in pregnant women with posttraumatic stress disorder: a pilot study. J Midwifery Women's Health 50:392-398

40. Leigh B, Milgrom J (2007) Acceptability of antenatal screening for depression in routine antenatal care. Aust J Adv Nurs $24: 14-18$

41. ACOG (2006) Committee Opinion No. 343: psychosocial risk factors. Perinatal screening and intervention. Obstet Gynecol 108:469-477

42. Austin MP, Priest SR, Sullivan EA (2008) Antenatal psychosocial assessment for reducing perinatal mental health morbidity. Cochrane Database Syst Rev CD005124

43. Muzik M, Marcus SM, Heringhausen JE, Flynn H (2009) When depression complicates childbearing: guidelines for screening and treatment during antenatal and postpartum obstetric care. Obstet Gynecol Clin N Am 36:771-788, ix-x

44. Grote NK, Bridge JA, Gavin AR, Melville JL, Iyengar S, Katon WJ (2010) A meta-analysis of depression during pregnancy and the risk of preterm birth, low birth weight, and intrauterine growth restriction. Arch Gen Psychiatry 67:1012-1024 